\title{
Normative data for the Balance Tracking System modified Clinical Test of Sensory Integration and Balance protocol
}

This article was published in the following Dove Press journal: Medical Devices: Evidence and Research

\author{
Daniel J Goble' \\ Harshan Brar' \\ Elise C Brown ${ }^{2}$ \\ Charles RC Marks' \\ Harsimran S Baweja ${ }^{3}$ \\ 'Department of Human Movement \\ Science, Oakland University, Rochester, \\ MI, USA; ${ }^{2}$ Public and Environmental \\ Wellness, Oakland University, Rochester, \\ MI USA; ${ }^{3}$ School of Exercise and \\ Nutritional Sciences, San Diego State \\ University, San Diego, CA, USA
}

Purpose: Force plate balance testing technology has traditionally been underutilized in clinical and research settings due to the high cost and lack of portability. A relatively new force plate called the Balance Tracking System (BTrackS) has been developed to overcome these barriers. BTrackS recently implemented the modified Clinical Test of Sensory Integration and Balance (mCTSIB) as a means of evaluating various sources of sensory information for postural sway control. The present study aimed to provide much needed normative data for the BTrackS mCTSIB protocol.

Materials and methods: Data from 604 healthy adults (308 women; 296 men) between the ages of 18 and 29 years were collected according to the BTrackS mCTSIB protocol. The protocol consisted of four, 20 -second static standing trials that manipulated relative contributions of the vision, proprioception and vestibular sensory systems through various eyes open/closed and foam/no foam conditions. Comparisons of men versus women and the impact of body size (ie body mass index) were determined so that relevant percentile rankings could be calculated.

Results: Analysis of variance showed an interaction between sex and task condition on the BTrackS mCTSIB $(p<0.001)$. This interaction indicated that women outperformed men on all conditions, but especially in the fourth trial where eyes were closed and standing was done on a compliant foam surface. Percentile rankings were calculated based on sex and BTrackS mCTSIB condition. No relationship was found between BTrackS mCTSIB results and body size. Conclusion: Normative data provided in this study are vital for establishing potential sensory feedback-based balance dysfunctions that may exist clinically or in laboratory settings. In addition, this data can aid in the tracking of changes over a rehabilitation period and/or the effectiveness of balance interventions.

Keywords: postural sway, BTrackS, reference values, force plate, center of pressure

\section{Introduction}

Standing balance can be defined as the ability to maintain upright posture on two feet without falling. Commonly viewed as a "motor" skill, standing balance actually relies to a large extent on three sensory systems: proprioception, vision and vestibulation. With respect to proprioception, balance information is relayed from skin, muscle and joint receptors providing, primarily, a representation of ankle joint position changes associated with body sway. ${ }^{1}$ The visual system, on the other hand, inputs information on head position relative to the surrounding environment, which can be used in a feedforward fashion to anticipate a loss in balance. ${ }^{2}$ In contrast, the
Correspondence: Daniel J Goble Department of Human Movement Science, Oakland University, Human Health Building 433 Meadow Brook Road, Rochester, MI 48309-4452, USA

Tel +I 2483648688

$\mathrm{Fax}+\mathrm{I} 2483648657$

Email dgoble@oakland.edu 
vestibular system serves as an internal conflict resolution system between the head and environment that is absent of external cues. Specifically, vestibular feedback provides an inertial gravitational reference system regarding head positional equilibrium. ${ }^{3}$

Measurement of postural sway is a common means of assessing standing balance in both clinical and laboratory settings. Postural sway is defined as a mechanism whereby sustained oscillatory motion occurs about a fixed postural position. ${ }^{4}$ The gold standard for measuring postural sway is via a medical device called a force plate. Force plates quantify postural sway using a metric called center of pressure (COP). COP can be calculated as the weighted average of forces applied to a force plate while standing upon it. Increased COP magnitude (ie greater postural sway) during quiet standing is found in many clinical conditions including multiple sclerosis, mild traumatic brain injury and stroke. ${ }^{5-7}$

Despite the established efficacy of force plate balance assessments, widespread use remains limited due to the high cost and lack of portability associated with most devices. Indeed, some traditional force plates cost in excess of $\$ 100,000$ and weigh greater than $50 \mathrm{~kg}$. These practical limitations were the impetus for developing the Balance Tracking System (BTrackS). BTrackS is a force plate balance testing solution that is both affordable $(<\$ 2,000)$ and portable $(<6.8 \mathrm{~kg})$ compared to its counterparts. BTrackS also promotes a number of time efficient and user-friendly protocols that accurately and reliably examine postural sway. ${ }^{8,9}$ One of the most popular protocols is the modified Clinical Test of Sensory Integration and Balance (mCTSIB). The MCTSIB is a derivative of the CTSIB, which was originally developed by Shumway-Cook and Horak. ${ }^{10}$ The CTSIB was created to evaluate the contribution of various sources of sensory information for balance.

The mCTSIB utilized by BTrackS objectively measures postural sway in each of four test conditions. The first condition is the "standard" test condition where all three sensory systems (ie proprioception, vision and vestibular) are available to assist in maintaining balance and, therefore, the smallest amount of postural sway is expected. In condition two, the eyes are closed to temporarily eliminate visual feedback, thus, increasing reliance on proprioceptive and vestibular systems. Since proprioception is more heavily utilized for balance than the vestibular system, the typical perspective is that this condition largely measures the contribution of proprioception to balance. ${ }^{3}$ In the third condition, the visual and vestibular systems are available, but the proprioceptive system is compromised by having the individual being tested stand on a compliant foam surface. If there is a difficulty maintaining postural sway in this condition, the visual system is typically implicated given its preference over vestibular feedback for balance. In the fourth condition, the eyes are closed and the individual stands on foam. In this case, the visual and proprioceptive systems are compromised, shifting reliance to the vestibular system as the primary sensory source used to maintain balance.

When assessing postural sway in either clinical or research settings, it is vital to have normative data to aid in the interpretation of results. Specifically, comparing test results to typical individuals assists in establishing whether or not a balance dysfunction exists. Additionally, normative data can be used to track positive and negative changes over the course of a rehabilitation or intervention period, helping establish effectiveness of a given treatment. Lastly, as it pertains to the BTrackS mCTSIB protocol, normative data can aide clinicians and researchers in determining the locus of a balance impairment (ie proprioceptive, visual and/or vestibular) through differential diagnosis.

The aim of the present study, therefore, was to establish the first set of normative data for the BTrackS mCTSIB protocol. This was accomplished by collecting BTrackS mCTSIB results from a large sample of healthy young adults and calculating true percentile rankings stratified by relevant sex and body size factors. It was hypothesized based on previous literature that significant differences in performance would be seen showing less postural sway (ie better balance) in women versus men. ${ }^{11}$ In contrast, it was expected that no relationship would be seen between body composition and MCTSIB results in any test condition. ${ }^{11}$ Overall, the results of this work provide an important resource for a growing cohort of clinical practitioners and research scientists using the BTrackS mCTSIB protocol.

\section{Material and methods}

\section{Participants}

In this study, data were collected from 604 healthy adults (308 women, 296 men) between the ages of 18 and 29 years (average $\pm \mathrm{SD}=22.7 \pm 2.8$ years). Participants gave written, informed consent and self-reported having no known balance impairment at the time of testing. Tests were conducted 
across multiple sites including health fairs, physical therapy clinics, fitness gyms, religious settings and educational institutions, among others. Ethical approval for this human subjects-based research was obtained through the institutional review board of Oakland University, and all procedures adhered to the Declaration of Helsinki and included written informed consent.

\section{Experimental setup}

The BTrackS mCTSIB was conducted using the BTrackS Balance Plate and BTrackS Assess Balance software (Balance Tracking Systems, San Diego, CA), which are depicted in Figure 1. The BTrackS Balance Plate is a lightweight force plate that is a registered medical device with the US Food and Drug Administration. The force plate measures $0.4 \times 0.6 \mathrm{~m}$ and has been ecologically validated in prior research. ${ }^{8}$ A converging body of evidence also exists showing that the BTrackS Balance Plate displays excellent accuracy and precision for the measurement of COP. ${ }^{12-14}$

BTrackS Assess Balance software (version 4) was installed on multiple computing devices (ie laptops and tablets), each running a full version of the Microsoft Windows operating system. The BTrackS Assess Balance software provided an on-screen interface for profile creation, test administration and result interpretation related to the mCTSIB. Due to the user-friendly nature of BTrackS Assess Balance, minimal training was required to learn how to administer the BTrackS mCTSIB protocol. In this case, test administrators became proficient after performing several tests under the guidance of an experienced user.

\section{Testing procedures}

Each testing session was conducted in an isolated space with limited distractions. To start each testing session, the BTrackS Balance Plate was leveled on a hard surface, using built-in height adjustable legs. The BTrackS Balance Plate was then connected to the computing device via a USB interface, which also provided power to the plate. Standardized instructions were read to participants, according to the following on-screen script:

You are about to perform a Clinical Test of Sensory Integration and Balance or CTSIB. The CTSIB consists of four, 20-second trials that measure your ability to control body sway when sensory feedback is systematically manipulated. For each trial, you will stand as still as possible on the BTrackS Balance Plate with your hands on your hips and feet shoulder width apart. You will hear a tone at the beginning and end of each trial. Your CTSIB results will be based on the Center of Pressure (COP) path length from the forces you place on the BTrackS Balance Plate during standing. Sensory feedback will be manipulated by having you close your eyes or stand on foam in some conditions.

In line with the above instructions, participants stood on the plate for four testing trials (one in each of four

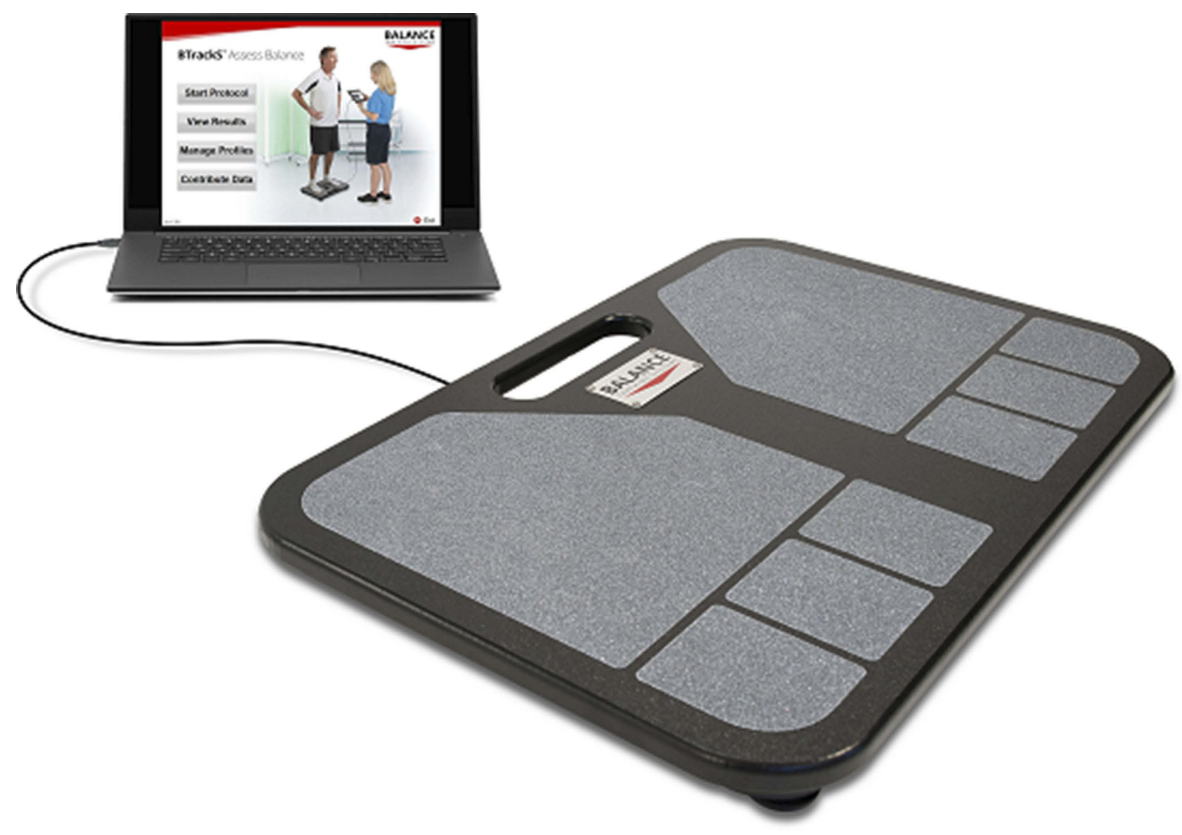

Figure I Testing materials for this study included the BTrackS Balance Plate (right) and BTrackS Assess Balance software running on a laptop (left). Abbreviation: BTrackS, Balance Tracking System. 
conditions) with their feet shoulder width apart and hands on their hips (Figure 2). The first trial (ie Standard condition, Figure 2A) was conducted with the eyes open while standing on the firm surface of the plate. The trial lasted for 20 seconds and began and ended with an auditory tone. After a minimal inter-trial delay ( $<10$ seconds), the second trial (ie Proprioception condition, Figure 2B) commenced with the participants eyes closed while standing on the firm surface. Following the second trial, trials three (ie Vision condition, Figure 2C) and four (ie Vestibular condition, Figure 2D) were conducted similar to trials one and two. However, for these trials the participants stood on
A

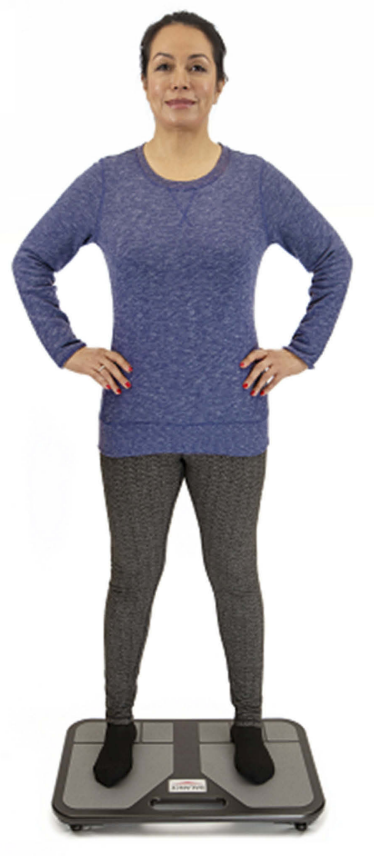

C

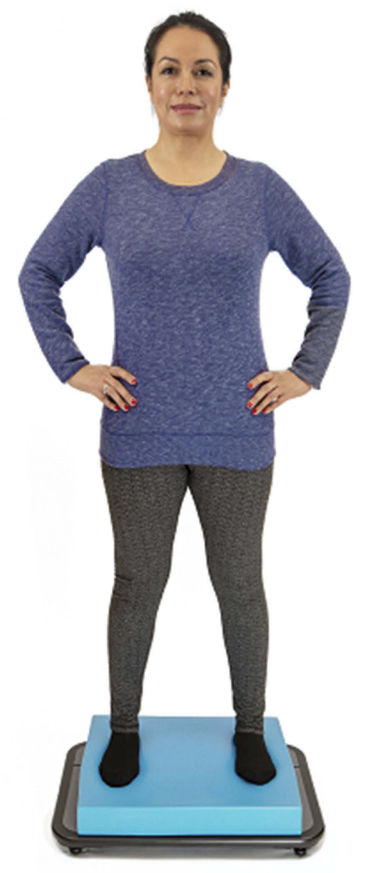

B

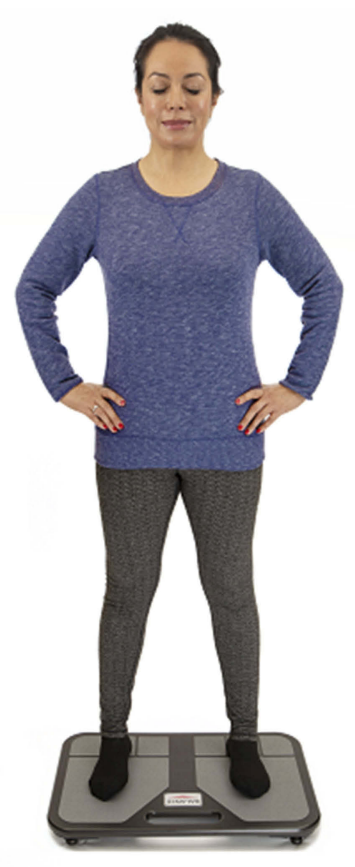

D

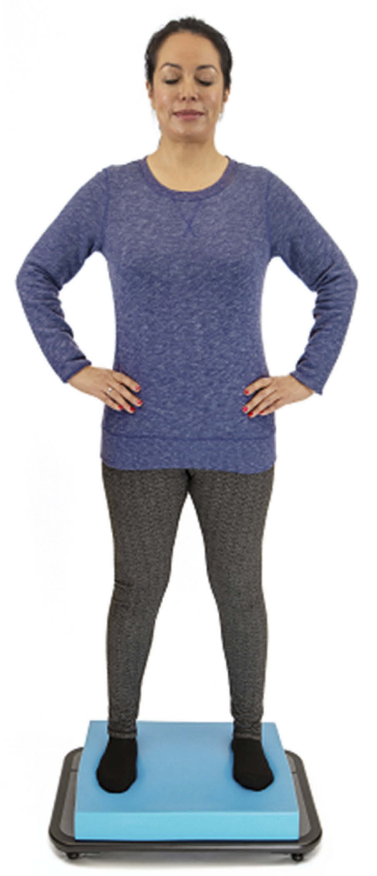

Figure 2 Testing positions for the BTrackS mCTSIB protocol. In all conditions, individuals stood as still as possible on the BTrackS balance plate with feet shoulder width apart and hands on hips. Condition I (A, Standard) was performed with eyes open while standing on the firm surface of the plate. Condition 2 (B, Proprioception) was performed with eyes closed while standing on the firm surface of the plate. Condition 3 (C, Vision) and condition 4 (D, Proprioception) were performed while standing on foam with eyes open and eyes closed, respectively. Image used with written informed consent of individual shown.

Abbreviations: mCTSIB, modified Clinical Test of Sensory Integration and Balance; BTrackS, Balance Tracking System. 
a piece of high-density foam. Specifically, in trial three the participants' eyes were open while standing on the foam, while in trial four, the eyes were closed while standing on the foam. Participants removed their shoes for all testing, however, previous research of similar static balance protocols has found no difference in COP with or without standard footwear. ${ }^{15}$

\section{Data analysis}

The result of each BTrackS mCTSIB trial was calculated by the BTrackS Assess Balance software, equivalent to the Total COP Path Length. Total COP Path Length is a proxy for postural sway magnitude whereby larger Total COP Path Length values are indicative of greater postural sway. ${ }^{9}$ Total COP Path Length was determined by first quantifying the point to point COP Path Length between successive time points according to the following formula:

$$
\begin{aligned}
\mathrm{COP} \text { path length }= & \left(\left(\mathrm{COP}_{\mathrm{x} 2}-\mathrm{COP}_{\mathrm{x} 1}\right)^{2}\right. \\
& \left.+\left(\mathrm{COP}_{\mathrm{y} 2}-\mathrm{COP}_{\mathrm{y} 1}\right)^{2}\right)^{0.5}
\end{aligned}
$$

where $\mathrm{COP}_{\mathrm{x} 2}$ and $\mathrm{COP}_{\mathrm{x} 1}$ are adjacent time points in the $\mathrm{COP}_{\mathrm{x}}$ (medial/lateral) time series and $\mathrm{COP}_{\mathrm{y} 2}$ and $\mathrm{COP}_{\mathrm{y} 1}$ are adjacent time points in the $\mathrm{COP}_{\mathrm{y}}$ (anterior/posterior) time series. The sum of all COP Path Lengths was then added together to get Total COP Path Length. The BTrackS Balance Plate sampling frequency is specified by the manufacturer at $25 \mathrm{~Hz}$ for a total of 500 data points in a 20s trial. Only Total COP Path Length was provided by the BTrackS Assess Balance software, thus, no other COP metrics were assessed.

Once all participant tests were completed, BTrackS mCTSIB results were de-identified and assimilated into a single database in preparation for statistical analyses. A quality inspection of the data was performed using a series of rules that determined improper use of the testing protocol, invalid demographics and testing outliers. Less than $1 \%$ of the original sample $(n=609)$ profiles was excluded. Following this pre-preprocessing step, the statistical effects of sex (men, women) and testing condition (standard, proprioception, vision, vestibular) on BTrackS mCTSIB, as well as the interaction between sex and condition (sex*condition), was performed using a two-factor analysis of variance (ANOVA) with repeated measures for condition. This analysis was conducted in SPSS (IBM, Armonk, NY) with significance determined at the $p<0.05$ level. Where significant effects were found, Tukey Honest Significant Differences (HSD) were used to determine significant differences between levels of a given factor. Lastly, based on the analyses for sex and condition, percentile rankings were calculated for the 1st, 10th, 20th, 30th, 40th, 50th, 60th, 70th, 80th, 90th and 99th percentiles according to the following formula:

$$
\text { Percentile ranking }=\mathrm{P} / 100(\mathrm{~N}+1)
$$

In this formula, $\mathrm{P}$ represents the percentile rank and $\mathrm{N}$ represents the number of $\mathrm{mCTSIB}$ results in the distribution of interest.

To quantify the influence of body size on postural sway, body mass index (BMI) was calculated for a large subset of individuals $(\mathrm{n}=225)$ according to the following formula:

$$
\text { BMI }=\text { Weight } / \text { Height }^{2}
$$

where weight was measured in Kilograms $(\mathrm{Kg})$ and Height was measured in meters (m). For each sex and condition grouping, linear regressions were then performed in SPSS between Total COP Path Length and BMI to obtain $\mathrm{R}^{2}$ values. $R^{2}$ values were between 0 and 1 , where 0 indicated no Total COP Path Length variance was explained by BMI (ie no relationship), and 1 indicated all variance was explained by BMI (ie perfect relationship).

\section{Results}

Based on the ANOVA, significant differences in Total COP Path Length were found due to sex $\left(F_{1,602}=31.3\right.$, $p<0.001)$, condition $\left(F_{4,599}=1728.8, \quad p<0.001\right) \quad$ and a sex*condition interaction $\left(F_{4,599}=8.1, p<0.001\right)$. These results are visually depicted in Figure 3 and demonstrate that women significantly outperformed men (ie had smaller Total COP Path Lengths) in each of the standard (Tukey HSD, $p<0.001$ ), proprioception (Tukey HSD, $p<0.001$ ), vision (Tukey HSD, $p<0.001$ ) and vestibular conditions (Tukey HSD, $p<0.001$ ). Further, the magnitude of sex differences increased across conditions, with the greatest sex difference found in the vestibular condition where both vision and proprioception were compromised.

Analysis of the relationship between body size and BTrackS mCTSIB performance showed that participant BMI explained very little ( $2 \%$ or less) of the variance in Total COP Path Length. Table 1 provides a sex by condition breakdown of these data. In light of these findings, percentile rankings were stratified only by sex and condition, and not body size. These percentile rankings are provided in "look-up" form in Table 2 (women) and Table 3 (men), and serve as a tool for understanding the results of an individual compared to a healthy young adult of the same sex for a given condition. For example, 


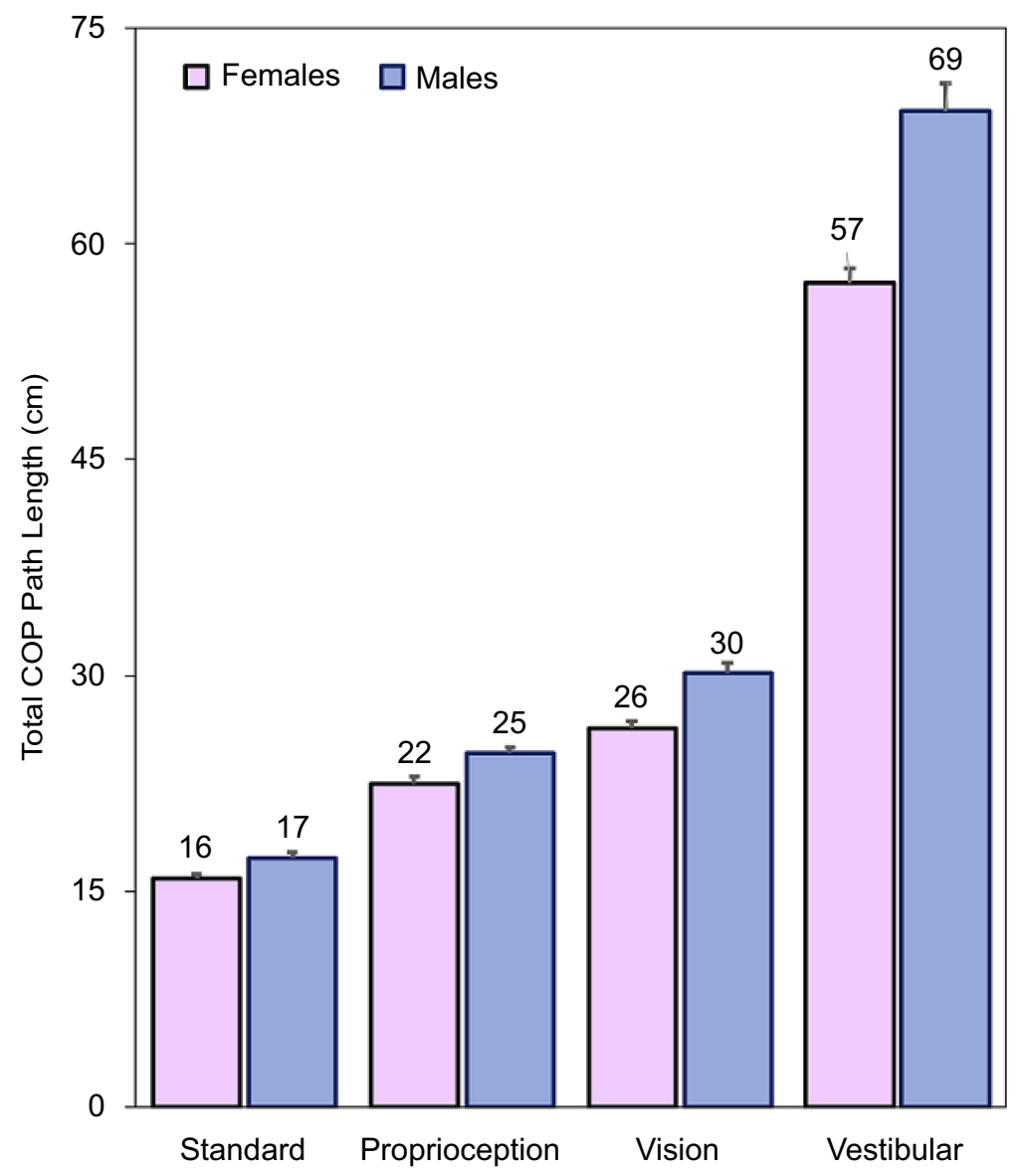

Figure 3 Mean (standard error) Total COP Path Length results for men and women in each BTrackS mCTSIB test condition.

Abbreviations: COP, center of pressure; BTrackS, Balance Tracking System; mCTSIB, modified Clinical Test of Sensory Integration and Balance.

Table I BTrackS mCTSIB variance explained by BMI for each test condition

\begin{tabular}{|l|l|l|l|l|}
\hline \multirow{2}{*}{$\begin{array}{l}\text { mCTSIB } \\
\text { Condition }\end{array}$} & \multicolumn{2}{|l|}{ Males } & \multicolumn{2}{l|}{ Females } \\
\cline { 2 - 5 } & $\mathbf{R}^{\mathbf{2}}$ & $\begin{array}{l}\text { Variance } \\
\text { explained }\end{array}$ & $\mathbf{R}^{\mathbf{2}}$ & $\begin{array}{l}\text { Variance } \\
\text { explained }\end{array}$ \\
\hline $\begin{array}{l}\text { Standard (eyes } \\
\text { open/firm) } \\
\text { Proprioception } \\
\text { (eyes closed/firm) } \\
\text { Vision (eyes open/ } \\
\text { foam) } \\
\text { Vestibular (eyes } \\
\text { closed/foam) }\end{array}$ & 0.001 & $0.1 \%$ & 0.020 & $2.0 \%$ \\
\hline
\end{tabular}

Abbreviations: BMI, body mass index; BTrackS, Balance Tracking System; mCTSIB, modified Clinical Test of Sensory Integration and Balance.

a man who has a Total COP Path Length of 59 on the vestibular condition is in the 40th percentile. This means his performance is as good as or better than $40 \%$ of healthy young adult men.

\section{Discussion}

The BTrackS Balance Plate is gaining popularity as an objective, portable and low-cost force plate for balance assessment. In this case, the present study sought to provide an initial set of normative data for the relatively new BTrackS mCTSIB protocol in a large sample of healthy young adults. Differences between women and men were seen across all conditions (ie standard, proprioception, vision, vestibular), with body size having little influence on performance. These findings informed the calculation of relevant percentile rankings for creating "look-up" tables clinicians and research scientists can use in a number of practical ways.

Lower postural sway (ie better balance) in women is a consistent result in the literature across a variety of testing protocols. ${ }^{11}$ Since there was very little relationship between body size and Total COP Path Length, the increase in sex differences across the various testing trials 
Table 2 BTrackS mCTSIB percentile rankings for women's Total COP Path Length results in each test condition

\begin{tabular}{|c|c|c|c|c|}
\hline \multirow[t]{2}{*}{ Percentile } & \multicolumn{4}{|l|}{ mCTSIB Condition } \\
\hline & $\begin{array}{l}\text { Standard (eyes open/ } \\
\text { firm) }\end{array}$ & $\begin{array}{l}\text { Proprioception (eyes } \\
\text { closed/firm) }\end{array}$ & $\begin{array}{l}\text { Vision (eyes open/ } \\
\text { foam) }\end{array}$ & $\begin{array}{l}\text { Vestibular (eyes closed } \\
\text { foam) }\end{array}$ \\
\hline Ist & 33 & 43 & 44 & 120 \\
\hline 10th & 22 & 31 & 36 & 80 \\
\hline 20th & 19 & 28 & 33 & 70 \\
\hline 30th & 17 & 25 & 30 & 63 \\
\hline 40th & 16 & 23 & 28 & 58 \\
\hline 50th & 15 & 21 & 26 & 54 \\
\hline 60th & 14 & 19 & 24 & 50 \\
\hline 70th & 13 & 18 & 22 & 46 \\
\hline 80th & 12 & 17 & 19 & 41 \\
\hline 90th & 10 & 14 & 16 & 38 \\
\hline 99th & 8 & 9 & 11 & 31 \\
\hline
\end{tabular}

Abbreviations: COP, center of pressure; BTrackS, Balance Tracking System; mCTSIB, modified Clinical Test of Sensory Integration and Balance.

Table 3 BTrackS mCTSIB percentile rankings for men's Total COP Path Length results in each test condition

\begin{tabular}{|l|l|l|l|l|}
\hline \multirow{2}{*}{ Percentile } & \multicolumn{2}{|l|}{ mCTSIB Condition } \\
\cline { 2 - 5 } & $\begin{array}{l}\text { Standard (eyes open/ } \\
\text { firm) }\end{array}$ & $\begin{array}{l}\text { Proprioception (eyes } \\
\text { closed/firm) }\end{array}$ & $\begin{array}{l}\text { Vision (eyes open/ } \\
\text { foam) }\end{array}$ & $\begin{array}{l}\text { Vestibular (eyes closed/ } \\
\text { foam) }\end{array}$ \\
\hline Ist & 34 & 53 & 71 & 151 \\
I0th & 26 & 35 & 44 & 97 \\
20 th & 22 & 31 & 36 & 83 \\
30 th & 19 & 28 & 34 & 74 \\
40 th & 17 & 26 & 30 & 69 \\
50 th & 16 & 23 & 28 & 63 \\
60 th & 15 & 21 & 25 & 59 \\
70 th & 14 & 19 & 22 & 54 \\
80 th & 12 & 17 & 21 & 50 \\
90 th & 11 & 15 & 18 & 45 \\
99 th & 9 & 11 & 14 & 31 \\
\hline
\end{tabular}

Abbreviations: COP, center of pressure; BTrackS, Balance Tracking System; mCTSIB, modified Clinical Test of Sensory Integration and Balance.

of the BTrackS mCTSIB suggests that this difference is somehow related to sensory feedback processing and/or difficulty of the testing condition. Specifically, the difference between men and women on the easiest of conditions (ie standard) was minimal when all sources of sensory information were highly available. In contrast, as one or more sources of sensory information were removed/ manipulated, women showed a greater resiliency in utilizing the remaining sensory sources to control postural sway.

The present findings are cross-validated by previously published normative data studies for the BTrackS Balance Test (BBT) protocol. ${ }^{11,16,17}$ The BBT protocol consists of four trials of static standing with hands on hips and feet shoulder width apart similar to the BTrackS mCTSIB. However, all BBT trials are performed with eyes closed in a manner that is equivalent to the proprioceptive condition (ie trial 2) of the BTrackS mCTSIB. For both the BBT and mCTSIB, young adult women outperformed men and body size had little correlation with performance. Percentile ranking comparisons between the BBT and its corollary (ie trial 2 ) in the mCTSIB are also highly consistent in magnitude and variance.

The present study was conducted at multiple sites, which may have introduced variability due to environmental factors. That said, the goal of this work is to provide a reference for clinicians and researchers in various field settings. In this case, the additional variance due to the 
diversity of testing location may actually strengthen the applicability of the results. Further, the present study is limited in that the sample collected may not be representative of the global population at large, as testing sites were primarily located in a single region of a single country (southeast Michigan, USA). Future work will address these limitations by expanding data collection efforts across a larger geographical area.

This study also fails to address known differences in postural sway measured by force plate devices that are seen with age. Work is already underway which will address this need for the clinical and research communities. Lastly, a final limitation of this study is that the decision to evaluate BMI was made post-hoc of balance testing and, thus, it was not possible to retroactively obtain values for all participants. That said, the sample collected was more than adequate for calculating ICC values, given that the recommended standard is at least 30 individuals per group. ${ }^{18}$ Indeed, our sample was more than three times greater than that with over 100 men and 100 women per group.

\section{Conclusion}

In conclusion, the percentile ranking information provided in this study is an important first step in helping a growing number of clinical and research professionals assess balance more effectively with the BTrackS mCTSIB protocol. Specifically, this information will allow for more adequate comparisons of results with healthy young adults to determine the existence of balance deficits as they pertain to sensory feedback sources. Through this enhanced diagnostic capability, it will be possible to better address the needs of individuals with balance deficits and provide them various balance interventions in a targeted, expeditious fashion.

\section{Acknowledgment}

The authors would like to formally acknowledge the multitude of research assistants who aided in the collection of this dataset.

\section{Disclosure}

DJG is eligible for royalties from a pending patent (OMB 0651-0032) related to the technology used in this study. In addition, he has an equity stake (stock options) in Balance Tracking Systems, Inc. This financial conflict of interest is mitigated by a management plan put in place by his academic institution to ensure the integrity of his research.
The authors report no other conflicts of interest in this work.

\section{References}

1. Nashner LM. Sensory feedback in human posture control [dissertation]. Massachusetts: Massachusetts Institute of Technology; 1970.

2. Nashner LM. Adaptation of human movement to altered environments. Trends Neurosci. 1982;5(10):358-361. doi:10.1016/ 0166-2236(82)90204-1

3. Nashner LM, Black FO, Wall C. Adaptation to altered support and visual conditions during stance: patients with vestibular deficits. JNeurosci. 1982;2(5):536-544. doi:10.1523/JNEUROSCI.02-05-00536.1982

4. Hellebrandt FA, Braun GL. The influence of sex and age on the postural sway of man. Am J Phys Anthropol. 1939;24(3):347-360. doi:10.1002/(ISSN)1096-8644

5. Morrison S, Rynders CA, Sosnoff JJ. Deficits in mediolateral balance control and the implications for falls in individuals with multiple sclerosis. Gait Posture. 2016;49:148-154. doi:10.1016/j. gaitpost.2016.06.036

6. Goble DJ, Manyak KA, Abdenour TE, Rauh MJ, Baweja HS. An initial evaluation of the BTrackS balance plate and sports balance software for concussion diagnosis. Int J Sports Phys Ther. 2016;11 (2):149-155.

7. Lee HH, Jung SH. Prediction of poststroke falls by quantitative assessment of balance. Ann Rehabil Med. 2017;41(3):339-346. doi:10.5535/arm.2017.41.3.339

8. Benedict S, Hinshaw JW, Byron-Fields R, Baweja HS, Goble DJ. Effects of fatigue on the BTrackS balance test for concussion management. Int J Athl Ther Train. 2017;22(4):23-28. doi:10.1123/ ijatt.2016-0086

9. Hearn MC, Levy SS, Baweja HS, Goble DJ. BTrackS balance test for concussion management is resistant to practice effects. Clin J Sport Med. 2018;28(2):177-179. doi:10.1097/JSM.0000000000000442

10. Shumway-Cook A, Horak FB. Assessing the influence of sensory interaction on balance: suggestion from the field. Phys Ther. 1986;66 (10):1548-1550.

11. Goble DJ, Baweja HS. Normative data for the BTrackS balance test of postural sway: results from 16,357 community-dwelling individuals who were 5 to 100 years old. Phys Ther. 2018;98(9):779-785. doi:10.1093/ptj/pzy062

12. O'Connor SM, Baweja HS, Goble DJ. Validating the BTrackS balance plate as a low cost alternative for the measurement of sway-induced center of pressure. $J$ Biomech. 2016;49 (16):4142-4145. doi:10.1016/j.jbiomech.2015.12.045

13. Richmond SB, Dames KD, Goble DJ, Fling BW. Leveling the playing field: evaluation of a portable instrument for quantifying balance performance. $J$ Biomech. 2018;75(25):102 107. doi:10.1016/j. jbiomech.2018.05.008

14. Goble DJ, Khan E, Baweja HS, O`Connor SM. A point of application study to determine the accuracy, precision and reliability of a low-cost balance plate for center of pressure measurement. J Biomech. 2018;71 (11):277-280. doi:10.1016/j.jbiomech.2018.01.040

15. Plom W, Strike SC, Taylor MJ. The effect of different unstable footwear constructions on centre of pressure motion during standing. Gait Posture. 2014;40(2):305-309. doi:10.1016/j.gaitpost.2014.04.189

16. Goble DJ, Baweja HS. Postural sway normative data across the adult lifespan: results from 6280 individuals on the balance tracking system balance test. Geriatr Gerontol Int. 2018;18(8):1225-1229. doi:10.1111/ggi.13452

17. Goble DJ, Rauh MJ, Baweja HS. Normative data for the BTrackS $S^{\mathrm{TM}}$ balance test concussion management tool. $J$ Ath Train. In press.

18. Cicchetti DV. Guidelines, criteria, and rules of thumb for evaluating normed and standardized assessment instrument in psychology. Psychol Assess. 1994;6((4):):284-290. doi:10.1037/1040-3590.6.4.284 


\section{Publish your work in this journal}

Medical Devices: Evidence and Research is an international, peerreviewed, open access journal that focuses on the evidence, technology, research, and expert opinion supporting the use and application of medical devices in the diagnosis, monitoring, treatment and management of clinical conditions and physiological processes. The identification of novel devices and optimal use of existing devices which will lead to improved clinical outcomes and more effective patient management and safety is a key feature of the journal. The manuscript management system is completely online and includes a very quick and fair peer-review system. Visit http:// www.dovepress.com/testimonials.php to read real quotes from published authors. 